Presented at the 1991 Particle Accelerator Conference, San. Fran., Calif.,5/6-9/9l

BNL- -46143

DE91 014027

\title{
INSTRUMENTATION and CONTROL of the AGS BOOSTER VACUUM SYSTEM*
}

\author{
J. Gabusi, J. Geller, H. C. Hseuh, P. Rosas, J. Sandburg, B.Sherget
P. Stattel and R. Zapasek \\ BROOKHAVEN NATIONAI LABORATORY \\ 40199 \\ UPTON, NEW YORK $; 1973$
}

\begin{abstract}
The AGS Booster is a synchrotron for the acceleration of both protons and heavy ions. A pressure of low $10^{-11}$ Torr is rquired for the acceleration of the partially stripped, low $\beta$. very heavy ions. This paper describes the power supplies and controls for this ultra-high vacurum system with the emphasis on the operation of the ion gauge system over long cable length and on equipment interiock.
\end{abstract}

\section{INTRODUCTION}

The recently completed AGS Booster [1] at Brookhaven is a small syachrotron of $200 \mathrm{~m}$ in circumference for the acceleration of protons and beavy ions. It is located between the existing $200 \mathrm{MeV}$ Linac, the Tandem Van de Graaff and the AGS. To minimize beam loss due to charge exchange [2] between the very heavy ions and the residual gas molecules, ultra-high vacuum of low 10-11 Torr is required. Various vacuum instrumentation is needed to produce, monitor and protect this ultra-high vacuum system. Due to the presence of high radiation levels in the Booster tunnel, all the power supplies and controls are located in the instrumentation building. The operation of these instruments over long cable length (up to $500 \mathrm{ft}$ ) and through computer interface are described in detail in this paper.

\section{VACUUM SYSTEMS}

The details of the Booster Vacuum System can be found in references 2 and 3. Major components relevant to the instrumentation are briefly described below.

Vacuum Chambers: The Booster ring has 48 half cells, isolatable with gate valves into seven vacuum sectors. A typical balf cell chamber is about $4.2 \mathrm{~m}$ long and consists of chambers for dipole, quadrupole, PUEs, sextupole, bellows and the transition with ports connecting to gauges, valves and pumps.

\footnotetext{
"Work performed under auspices of the U. S. Depl. of Energy.
}

Vacuum Pumps: The designed ring vacuum was achieved by . a combination of titanium sublimation pumps and ion pumps. Fifty five titanium cartridges with three filaments each are mounted in the UHV bodies to pump active gases. The nongetterable gases such as methane and argon are removed by diode ion pumps. Ion pumps and non-evaporable getter pumps are used to pump the beam transport lines. Portable turbopump stations were used during pumn down, bakeout and conditioning of the ring and lines.

Vacuum Gauges: Pirani gauges and Bayard-Alpert type nude ionization gauges are used in the Booster for measuring total pressures. Quadrupole type residual gas analyzers (RGA) are used in ring sectors for monitoring the partial pressures.

Bakeont: The vacuum chambers and the components within are designed to be insitu bakeable to $300^{\circ} \approx$. Custom heating blankets and E-type thermocouples (TCs) were installed around the chambers before assembling into magnets. The blankets were terminated to half-cell contactor boxes, which were then connected along with the TCs to the Programmable logical controller (PLC) carts before the bake. The PC based PLC initiates and maintains control over the programmed bake cycles, and alarms the operators when abnormal or failure conditions occur.

\section{VACUUM INSTRUMENTATION and CONTROL}

The vacuum instrumentation consist of the power supplies: for ion pumps and titanium pumps, controllers for vacuum gauges and valves, and the computer systems. The instrumentation laycut of a typical vacuum sector is depicted in Figure 1. The gauge controllers comminicate with the device controllers (D/C) through RS232 lints. The ion pump power supplies and valve controllers are linked to the $D / C$ s through IEEE-488 compatible Datacon interface cards. The D/Cs communicate vith the Apollo system via a station drop.

Titanium Pun, I-wer Supply: The titanium pump power supplies degas the titanium flaments during pumpdown and 


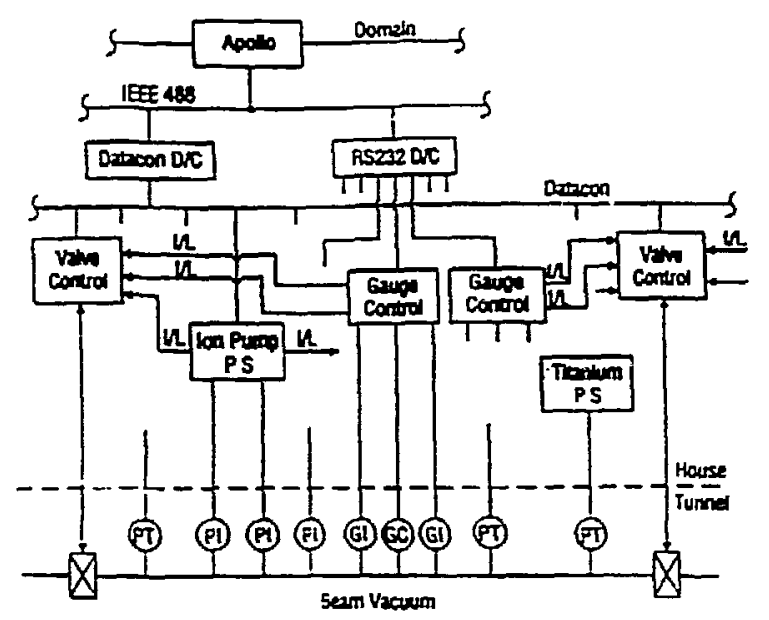

Figure 1

The Layout of Instrumentation of Typical Vacuum Sectors

bakeout, and sublime titanium to the UHV bodies when the aris3. These supplies consist of SCR based contrcllers which power and regulate the sublimation rate through the constant curreat mode. The SCRs step up the filament current through transformers locatod near the cartridges. By calibrating the gain and offset pots of each SCR versus the transformet ratios, current stability of better than $\pm 2 \%$ is achisved during sublimation at $48 \mathrm{~A}$. The sublimatica rate if approximately $1 \mathrm{mg} / \mathrm{min}$. One gram of titanium per filament is stailable by using this constant current mode.

lon Pump Power Supply: The power supplies, using ferroresonant transformers, develop potentials up to $5 \mathrm{KV}$ and are current limited to $200 \mathrm{~mA}$. Both voltage and current are measured for pressure monitoring and for diagnostics. Current down to 10 uA can be reliably measured through the linear and $\log$ amplifiers. The measured current and voltage are converter to frequencies and fed to Datacon interface cards for computer monitoring and display. Interlock and status signals, for controlling sector valves and other equipment, are opto-coupled for ground isolation.

Vacuum Monitoring: The vacuum is monitored by Pirani gauges, Bayard-Alpert type ion gauges, ion pump current and RGAs. The Pirani gauges cover from atmosphere to $1 \times 10^{-4}$ Torr. The ion pump current can be used reliably for pressure measurement down to $10^{-9}$ Torr. the ion gauges, having a thin collector of $0.05 \mathrm{~mm}$ diameler and an X-ray limit of $5 \times 10^{-12}$ Torr [4], can measure vacuum from $10^{-3}$ Torr to high $10^{-12}$ Torr.

Commercial vacuum process controllers (VPCs) are used to power, monitor and interlock the gauges, the ion pumps and the titanium pumps. The VPCs have process control channels utich can be assigned either to ion gauges or Pirani gauges.
The Pirani gauges interlock the turning-on of the ion gauges, the ion pumps and the titanium pumps in the same sectors. The ion gauges are mainly used to interlock sector valves and other equipment.

To overcome losses over long cable runs (up to 500'), large gauge wires and modified transformers in the VPCs with 40\% higher output voltage are used to heat the filaments, especially during electron bombardmeat (EB) degassing. Figure 2 gives the measured filament heating power during EB degassing for two cable length and two types of filaments. The standard transformers can only degass thoria-casted iridium filaments over long cable leagth. The modified transformers allow degassing of tungsten filament up to 35 watts over 500 feet $\# 12$ AWG cables.

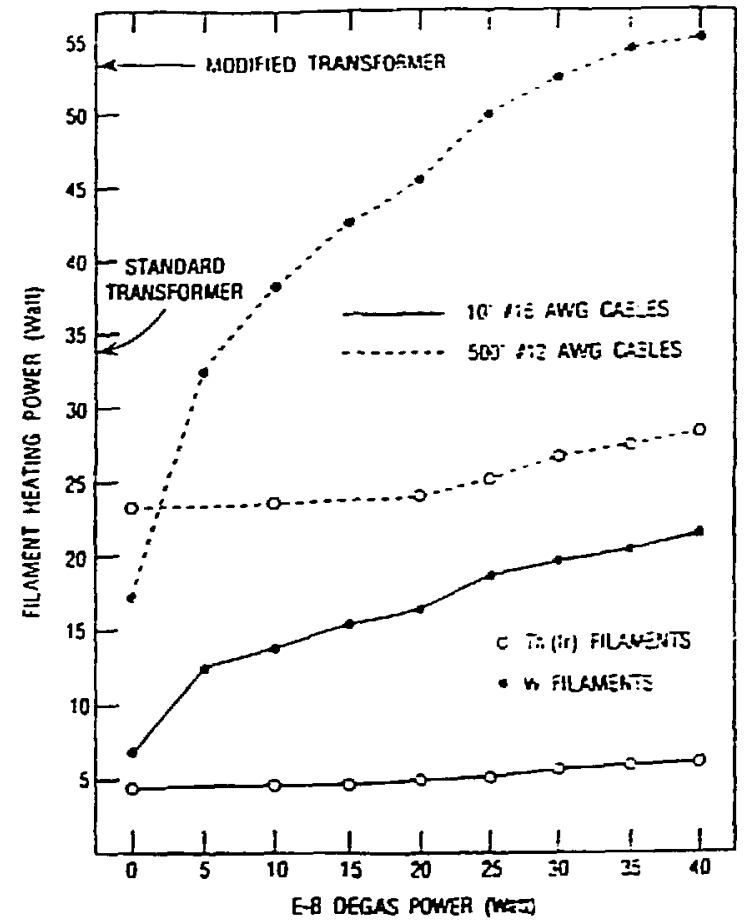

Figure 2

Filament Heating Power Versus the Electron Bombardment Degassing Power Over Different Cable Length for Two Types of Ion Gauge Filaments

At low $10^{11}$ Torr vacuum, the collector curreat is only a few picoampere and is susceptible to noise pick-up such as the electricai-magnet interference (EMI) of power supplies and the radio-frequency interfenence (RFI) of wccelerating cavities. The collector conxial cable is a potential source of noise especially over long length, resulting in erratic pressure readings. Tests of noise pick-ups over long cable runs in the AGS ring during machine operation have been carried out in the last few years. The results are summarized in Table 1 . Regular RG-59 coax, with effective shielding around $90 \%$, has microphonics of over $100 \%$ at mid $10^{\prime \prime}$ Torr. To minimize 
EMI/RFI, Beldon 9311 cable is used. This cable has microphonics of approximately $30 \%$ at mid $10^{\prime \prime}$ Torr and is acceptable for Booster vacuum operation. This coax features 100\% shield coverage, a foil/braid outer shield, a polyethylene dielectric of $26 \mathrm{pf} / \mathrm{ft}$, and good DC performance. The grid and filament wires (4 each., 12 AWG) are also placed in 2 single twisted shielded, Iow smoke and radiation retardant jacket.

\section{Table 1 Noise Pick-Up of Long Coaxial Cables}

\begin{tabular}{|c|c|c|}
\hline Cable Troe & Vacuum/Envinonment* & Mrantarix \\
\hline$\overline{R G 59} \mathrm{NU}, 1000^{\circ}$ & $5 \times 10^{-11}$ in Lab & $+20 \%-20 \%$ \\
\hline RG $59 \mathrm{NU}, 650^{\circ}$ & $5 \times 10^{-11}$ in $A G S$ w/ RF & $+2005-100 x$ \\
\hline RG 59 AU, $650^{\circ}$ & $5 \times 10^{-11}$ in AGS w/o RF & $+25 \%-25 \%$ \\
\hline RG $59 \mathrm{NU}, 650$ Triexial & $3 \times 10^{-11}$ in $A G S w / R F$ & $+30 \%-30 x$ \\
\hline Beldon $9223,500^{\circ}$ & $4 \times 10^{-11}$ in $A G S w / R F$ & $+100 x-50 x$ \\
\hline Beldon $9311,500^{\circ}$ & $2 \times 10^{-11}$ in $A G S w / R F$ & $+50 x-50 x$ \\
\hline Beldon $9311,500^{\circ}$ & $2 \times 10^{-11}$ in AGS w/o RF & $+30 \%-30 \%$ \\
\hline Beldon $9311,500^{\circ}$ & $5 \times 10^{-11}$ in AGS $w /$ RF & $+30 x-20 x$ \\
\hline
\end{tabular}

*Vacuum in Torr; " $w / R^{\prime}$ " = magnets and cavities ramping: "in AGS" = cabling from power supply house to UHV chamber in AGS tunnel.

One RGA nead is installed at each ring vacuum sector and will be powered with portable RF box and controller to measure the residual gas composition and for trouble shocting. The RGAs have proven to be very powerful tools during the conumissioning of vacuum sectors. It identified the presence of leaks or contaminants immediately even at vacuums as low as $10^{-11}$ Torr level.

Valve Control and Interlock: The beam vacuum is protected by sector valves. A fault detected by any two ion pumps or ion gauges in the same sector will cause the valves at both ends of the sector to close, thus minimizing the loss of vacuum in adjacent sectors. This voting scheme minimizes false triggering due to noise or malfunctioning of individual devices. A fast closing valve with a closing time of less than $15 \mathrm{msec}$ is also used between the Liriac and the Booster to protect the Booster ultra-high vacuum ring from catastrophic failure. To prevent beam damage to the valves, the valve closing signals will trigger the fast beam interrupt system located at the ion source within $350 \mu \mathrm{sec}$. Auxiliary interlock $\mathrm{I} / \mathrm{O}$ s in the valve controllers also allow for interlocking other valves or equipment.

The entire vacuum instrumentation system, except for equipment in the Booster-to-AGS line, is in operaiion. A few false closings of the valves occurred during beam commissioning and were found to be caused by ion gauge shut downs. This problem is under investigation.

\section{REFERENCES}

1. W.T. Weng, "Contruction and Early Commissioning Results of the AGS Booster",ibid.

2. H.C. Hseuh, et. al., IEEE 2569-0, p 574, (1989).

3. H.C. Hseuh, Vacuum, 41, 1903 (1990)

4. H.C. Hseuh and C. Lanni, J. Vac. Sci., Technol.,AS, 3244 (1987).

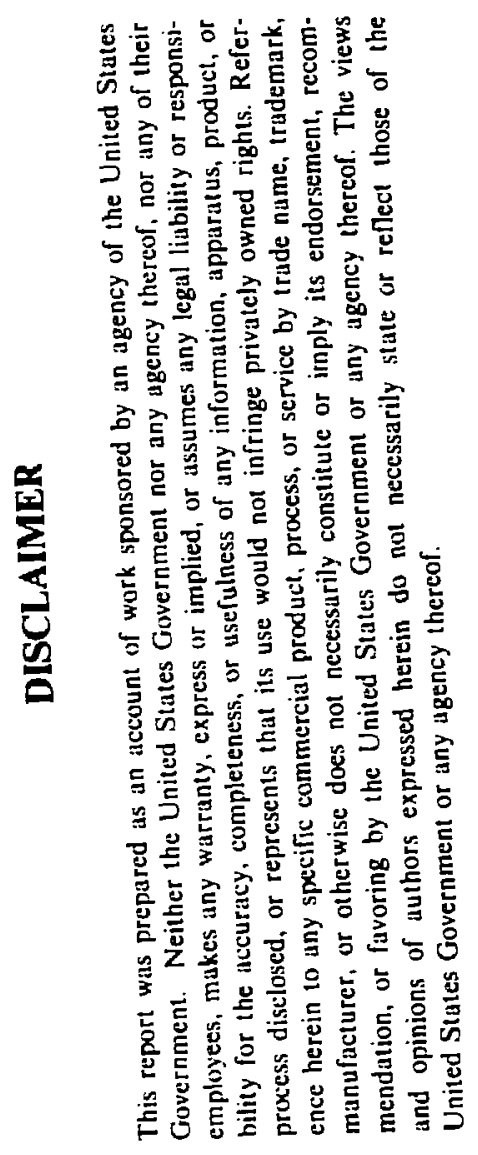

